\title{
A dimensão temática da pesquisa em organização do conhecimento: uma análise de domínio dos congressos nacionais e regionais da ISKO (Brasil, Espanha e América do Norte)
}

\author{
El ámbito temático de la investigación en organización del conocimiento: \\ un análisis de dominio de las conferencias nacionales y regionales de ISKO (Brasil, España y América del Norte)
}

The subject dimension of knowledge organization research: a domain analysis of the Brazilian, Spanish and North American ISKO chapters' conference

\section{José Augusto Chaves Guimarães, André Ynada dos Santos, Gilberto Gomes CÂNDIDo (1), Fabio Assis PINHO (2)}

(1) Universidade Estadual Paulista, Av. Hygino Muzzi Filho, 737, Marília, 17525-900, SP, Brasil, guima@marilia.unesp.br; (2) Universidade Federal de Pernambuco, Avenida da Arquitetura, s/n, Cidade Universitária, Recife, 50740-550, PE, Brasil, fabiopinho@ufpe.br.

\section{Resumen}

Se presentan los resultados una investigación exploratoria del análisis de dominio de los artículos publicados en las conferencias de los capítulos de la International Society for Knowledge Organization (ISKO) de Brasil, España y América del Norte, con el objetivo de identificar los temas y características de la colaboración científica como base para futuros estudios de tendencias y para facilitar la expansión de la participación de cada comunidad. Los resultados mostraron que los capítulos de ISKO de Brasil, Estados Unidos e Ibérico están desarrollando una trayectoria de la producción científica efectiva, con puntos focales temáticos como las cuestiones epistemológicas, por ejemplo, sin dejar de lado las preocupaciones de orden más regional. En este contexto, la presencia de varios investigadores en más de un espacio (o los tres) revela una preocupación y un compromiso con el diálogo académico sobre la organización del conocimiento, especialmente de la Universidad Estadual Paulista (UNESP, Brasil), la Universidad de Wisconsin-Milwaukee (UWM, Estados Unidos), y la Universidad Carlos III de Madrid (UC3M, España) que, además, están intensificando su colaboración en publicaciones conjuntas.

Palabras clave: Organización del conocimiento. Análisis de dominio. Productividad científica. Redes de colaboración. ISKO. Brasil. Estados Unidos. Iberia.

\section{Introdução}

A organização do conhecimento, termo inicialmente utilizado por Bliss em suas obras The organization of knowledge and the system of sciences, de 1929, e The organization of knowledge in libraries, de 1933, é considerada, nos dias de hoje, uma área nuclear na Ciência da Informação, estando diretamente relacionada

\begin{abstract}
This article is the result of a domain analysis of the articles published in the congresses of ISKO in Brazil, Spain and North America, aiming at identifying the themes and scientific collaboration characteristics, as an input for future studies on research trends and promoting the growth of the participation in each community. So it was characterized as an exploratory research focused on the analysis domain. The results showed that the Brazilian, North American and Iberian ISKO Chapters have been building a large space of scientific production with some convergent subjects like the epistemological questions, but also considering the regional specific ones. In this vein, the presence of researchers in the three studied academic spaces reveals a concern on establishing dialogical basis in KO research, whose main research institutions are São Paulo State University (UNESP, Brazil), University of Wisconsin Milwaukee (UWM, United States) and University Carlos III de Madrid (UC3M, Spain), which have deeply intensified their collaboration in joint publications.
\end{abstract}

Keywords: Knowledge organization. Domain analysis. Scientific productivity. Collaboration networks. ISKO. Brasil. Estados Unidos. Iberia.

com o armazenamento, recuperação e preservação da informação (Broughton, 2008) ou, em outros termos, com a organização de um "conhecimento em ação", destacado por Dahlberg (1993, p. 214) - trabalhando-se aqui com o conhecimento socializado, registrado e divulgado cuja organização e representação será desenvolvida de modo que, a partir dele, possa ser 
gerado novo conhecimento (Barité, 2001; Guimarães, 2001).

Situada na confluência das Ciências Cognitivas com a Ciência da Informação, a Ciência da Comunicação, a Matemática e a Ciência da Computação, a Organização do Conhecimento pode ser caracterizada pela sua natureza tanto científica (por estar sujeita à análise, reflexão e aplicação de fundamentos e métodos científicos) quanto social (dado seu caráter aplicado) (García Marco, 1997; Miranda, 1999).

Miranda (1999, p. 69), por sua vez, ressalta o fato de que a organização do conhecimento se apresenta como "disciplina científica, inter e transdisciplinar" aspecto que vai ao encontro da concepção de García Marco (1995, p. 87) para quem, dada a natureza interdisciplinar da organização do conhecimento, podem ser observados sete paradigmas sobre os quais esse campo, sob a ótica do referido autor, sustenta: conservacionista, fisicalista-informacional, semiótico-linguístico, lógico-matemático, cognitivo, econômico-empresarial e sociológico.

Tentando oferecer subsídios ao processo de construção epistemológica da área, Barité (2001, p. 42-53) propõe um conjunto de premissas, nas quais destaca o conhecimento, enquanto produto, necessidade e dínamo social, que realiza-se a partir da informação (e, ao socializar-se, nela novamente se transforma) e possui uma estrutura e um processo de comunicação abertos. Nesse sentido, seu registro e socialização ocorrem em documentos (conjunto organizado de dados disponíveis), e se expressa por meio de conceitos que têm seu processo de organização operado por meio de sistemas de conceitos (com fins científicos, funcionais ou de documentação), pautados por uma tônica artificial, provisória e determinista, com o fim último de promover seu melhor aproveitamento individual e social.

Tal aspecto pode ser observado na concepção de Esteban Navarro e García Marco (1995), pois esse campo apresenta natureza tridimensional uma vez que centra seus esforços nos princípios, métodos e instrumentos para a gestão do conhecimento humano, a partir de uma tripla perspectiva: sua representação, sua organização e sua comunicação documental.

Enquanto espaço científico, a organização do conhecimento encontra materialização na International Society for Knowledge Organization ISKO, criada na Alemanha, em 1989, por iniciativa de Ingetraut Dahlberg e Dagobert Soergel, para congregar pesquisadores em organização do conhecimento, com o intuito de promover o avanço teórico e aplicado dessa área em âmbito internacional, e em uma atividade interdisciplinar de áreas como Ciência da Informação, Linguística, Filosofia, Ciência da Computação entre outras.

No que tange às atividades científicas, a ISKO promove bienalmente as conferências internacionais (Darmstadt, 1990; New Delhi, 1992; Copenhagen, 1994; Washington, 1996; Lille, 1998; Toronto, 2000; Granada, 2002; Londres, 2004; Viena, 2006; Montréal, 2008; Roma, 2010; Mysore, 2012 e Cracóvia, 2014) (1). Além disso, a ISKO publica a revista bimestral Knowledge Organization e a série Advances in Knowledge Organization.

Tendo em vista a dimensão da ISKO enquanto organização, sentiu-se a necessidade do desenvolvimento de capítulos nacionais ou regionais, de forma a propiciar uma discussão mais verticalizada das questões de OC, e que atendam às peculiaridades sócio-político-culturais dos distintos entornos. Desse modo, a ISKO conta hoje com os seguintes capítulos ativos: Alemanha/Áustria/Suíça, Brasil, Canadá/Estados Unidos, China, Espanha, França, Índia, Irã, Itália, Maghreb (Marrocos/Argélia/Tunísia), Polônia e Reino Unido.

O capítulo espanhol da ISKO foi criado em 1990, por iniciativa de Emília Currás, tendo por intuito promover um espaço de interlocução acadêmica interdisciplinar, através de diálogos com as áreas de Lógica, Teoria de Sistemas, Ciência da Organização, Psicologia, Teoria da Ciência, Ciência da Computação, Semiótica, Linguística e Filosofia, especialmente a Epistemologia. As conferências do capítulo espanhol da ISKO vêm ocorrendo desde 1993, em anos ímpares, na seguinte sequência: Madri, 1993; Getafe, 1997 e 1999; Alcalá de Henares, 2001; Salamanca, 2003; Barcelona, 2005; Leon, 2007; Valencia, 2009; Ferrol, 2011 e, em 2013, em uma iniciativa de integração ibérica, teve lugar na cidade do Porto, em Portugal.

Por isso, o objetivo desta pesquisa foi identificar as características temáticas e de colaboração científica como subsídio para estudos futuros de tendências e expansões de participação de cada comunidade. De maneira mais específica, objetivou-se identificar temas e subtemas e categorizá-los, verificar temas predominantemente comuns e os regionalizados; verificar diálogos e interlocuções a partir dos autores e instituições.

\section{Metodologia}

Esta pesquisa configurou-se como exploratória e desenvolveu-se a partir do conjunto de atas 
dos congressos dos capítulos brasileiro, espanhol e norte-americano da ISKO, relativamente aos dois últimos congressos realizados $(2011 \mathrm{e}$ 2013). A escolha dos referidos capítulos deu-se no intuito de se fazer uma triangulação entre Europa, América do Norte (que possui um único capítulo), América do Sul (que possui apenas o capítulo brasileiro) e Europa (que possui os capítulos britânico, espanhol, francês, alemão, italiano e polonês), sendo que a escolha do capítulo espanhol para representar a Europa deve-se ao fato de ter sido um dos mais antigos (criado em 1990) e que guarda uma forte trajetória de interlocução com os demais. Dessa forma, e como o capítulo brasileiro realizou até então apenas dois congressos, optou-se por analisar comparativamente os dois últimos congressos realizados em cada capítulo selecionado.

A partir dessa delimitação, a pesquisa centrouse na abordagem da análise de domínio que, no âmbito internacional da Ciência da Informação, vem sendo, tradicionalmente, trabalhada em termos teóricos e aplicados por Hjørland e Albrechtsen (1995), Moya Anegón e Herrero Solana (2001), Hjørland (2002, 2004), Tennis (2003) e Smiraglia (2011), dentre outros, uma vez que, constitui uma importante abordagem para caracterização e avaliação da ciência, na medida em que permite identificar as condições pelas quais o conhecimento científico se constrói e se socializa.

O conceito de análise de domínio foi utilizado, inicialmente, por Neighbors, em 1980, na área de Ciência da Computação, no intuito de identificar elementos (operações, objetos e as relações deles decorrentes) que especialistas de um dado domínio consideram como significativos para suas atividades (Kerr, 2003). A partir dos anos 90 do século $\mathrm{XX}$, a análise de domínio passou a ser objeto de atenção mais específica da Ciência da Informação, a partir de estudos metateóricos (Ritzer, 1991; Vickery, 1997; Hjørland, 1998; Bates, 2005; Tennis, 2008) de modo a que se pudesse melhor explicar o substrato teórico e metodológico da própria área a partir da explicação de como se constrói, se caracteriza e se comporta um domínio.

Considerado por Hjørland e Albrechtsen (1995, p. 401) como uma "comunidade de pensamento ou comunidade discursiva que integra a divisão social do trabalho" o domínio se caracteriza pela delimitação de um dado conhecimento que se constrói por meio da interação de conceitos, estes também entendidos como unidades de conhecimento (Thellefsen e Thellefsen, 2004). Desse modo, o significado de um conceito está sempre em referência a um dado domínio e que um mesmo conceito comporta-se de formas distintas se analisado em outro domínio de conhecimento. Chega-se, assim, a "uma área de especialidade, um conjunto literário ou um grupo de pessoas trabalhando juntas numa organização" (Mai, 2005, p. 605), ou, ainda, a "uma área de conhecimento, atividade, interesse ou aplicação com limites definidos" (Llorens, 2004).

Tennis (2008) representa um domínio por duas dimensões (que podem ser encaradas como ordenada e abscissa) que se referem, respectivamente, à extensão ou amplitude de cobertura do domínio (áreas de modulação) e à intensão ou profundidade de abordagem (áreas de especialização). Essas dimensões se aplicam, especialmente, às possíveis abordagens da análise de domínio propostas por Hjørland (2002): Produção de obras de referência, Construção de linguagens de indexação, Indexação e recuperação da informação, Estudo de usuários, Estudos bibliométricos, Estudos históricos, Estudos de gêneros/tipologias documentais, Estudos epistemológicos e críticos, Estudos terminológicos, Comunicação científica, Cognição científica, conhecimento especializado e inteligência artificial.

Relativamente aos procedimentos de pesquisa, identificaram-se, em cada ata de congresso analisado, os temas, os subtemas, os assuntos, a autoria e a procedência geográfica. Em termos de autoria, identificaram-se os autores mais produtivos, sua procedência geográfica e as colaborações científicas. Sobre as temáticas, realizaram-se análises em dois níveis: a) em âmbito macro, a partir dos temas oficiais e subtemas (nesse caso, verificando os títulos dos artigos) de cada evento e as comparações entre eles e, b) em âmbito micro, a partir das palavras-chave, verificando os assuntos de maior predominância. Por fim, os dados foram comparados de modo a identificar especificidades e questões que levaram a diálogos ou interlocuções.

\section{Apresentação dos dados}

Primeiramente, convém mencionar que cada evento tende a possuir um tema oficial. A partir dele identificaram-se os subtemas que, para aqueles que não os possuíam, foram determinados a partir da análise dos títulos dos artigos. Por conseguinte, têm-se os assuntos que, por sua vez, emanam das palavras-chave atribuídas aos artigos. Por isso, foram consideradas essas três esferas para a realização da análise temática, ou seja, tema, subtema e assunto.

No evento ISKO-Brasil de 2011, o tema versou sobre os "Desafios e perspectivas científicas para a organização e representação do conhe- 
cimento". No evento da ISKO-Brasil em 2013, o tema foi sobre a "Complexidade e organização do conhecimento desafios do nosso século". Ao analisar esses dois anos identificou-se um total de 81 trabalhos distribuídos nos seguintes subtemas: a) dimensão epistemológica da ORC: 21 trabalhos; b) dimensão aplicada da ORC: 25 trabalhos; c) dimensão social, cultural e política da ORC: 24 trabalhos; d) pesquisa em ORC no Brasil: 7 trabalhos; e) visibilidade científica e interlocução internacional em ORC: 6 trabalhos.

Os artigos apresentados no subtema da dimensão epistemológica apresentaram os assuntos: a intersecção da epistemologia e a organização do conhecimento, as relações da ontologia e a organização do conhecimento, a produção científica, as concepções de análise documental e análise documental de obras de ficção, o conhecimento arquivístico e uma reflexão sobre o campo científico da Ciência da Informação, os panoramas de pesquisa, as contribuições interdisciplinares, as superestruturas documentais, a produção científica o mapeamento conceitual, reflexões sobre o processo de representação e suas linguagens.

No subtema da dimensão aplicada da ORC encontram-se assuntos sobre a construção, uso e consolidação de ontologias, folksonomias e vocabulários controlados, sistemas de classificação, metodologias e prática de indexação, assunto, linguística aplicada, modelos conceituais, intersecção das Ciências da Informação e da Computação, com ênfase no processamento semântico, e descrição arquivística.

No subtema da dimensão social, cultural e política têm-se assuntos como: pesquisas sobre mapeamento imaterial e sua representação, questões éticas, de identidade cultural e religiosa na representação do conhecimento e os problemas contemporâneos da representação do conhecimento, em domínios específicos, e as relações entre teoria e prática.

No derradeiro subtema da visibilidade científica encontram-se: os desafios sobre a internacionalização da área, bem como as reflexões sobre como as interlocuções científicas ocorrem.

O congresso da ISKO Espanha de 2011 comemorou os 20 anos do capítulo espanhol da IS$\mathrm{KO}$, sendo que o evento de 2013 teve por tema central: "Informação e/ou conhecimento: as duas faces de Jano", totalizando 124 trabalhos, com os seguintes subtemas: a) História da OC na Espanha: 6 trabalhos; b) Produção científica em OC na Espanha: 6 trabalhos; c) Questões teóricas e metodológicas da OC: 48 trabalhos; d) Desafios e perspectivas da OC em um âmbito disciplinar complexo: 13 trabalhos; e) Interope- rabilidade, acesso e compartilhamento da informação: 29 trabalhos; f) Igualdade e diversidade em um âmbito de informação global e local da OC: 7 trabalhos; e g) Organização da informação para a gestão do conhecimento: 15 trabaIhos

No subtema sobre os aspectos históricos destacaram-se assuntos como: o futuro da ISKO, classificação e, pesquisa científica. Em relação ao subtema da produção científica destacaramse os assuntos: rede social, colaboração científica, análise bibliométrica, análise de domínio, referentes teóricos, linguagem de indexação e base de dados.

As questões teóricas e metodológicas que integram o universo epistemológico da área se revelaram por meio de assuntos relativos aos sistemas de organização do conhecimento, à teoria do círculo de Bakhtin, à análise conceitual, e às relações interdisciplinares com a semiótica, à terminologia, à filosofia da linguagem e à cognição.

Os modelos e métodos da área conjugaram os assuntos: análise conceitual, elaboração de índices, arquitetura da informação, normalizações e práticas discursivas, análise de domínio, modelagem conceitual, método diplomático e aplicações de linguagens de indexação a contextos específicos.

Sobre os desafios e às perspectivas da organização do conhecimento, abordaram-se assuntos de cooperação internacional, do controle de autoridade, da usabilidade, dos dilemas éticos, dos padrões nas linguagens documentais e da engenharia do conhecimento na atualidade.

No âmbito da normalização e interoperabilidade foram destacados os seguintes assuntos: artes digitais, vocabulário de indexação e tesauros, classificação decimal universal, vocabulários estruturados e controlados, revistas científicas, bibliotecas digitais, padrões para cordel, repositórios, padrões para documentos arquivísticos digitais, descrição arquivística e fontes de informação. Tais aspectos se refletiram, ainda, em assuntos relativos ao compartilhamento da informação em comunidades virtuais, com ênfase na web 2.0, nas políticas e funcionalidades da classificação, no acesso aberto à informação, na governança, na informação acadêmica e na construção de repositórios em diferentes ambiências.

O subtema da igualdade e da diversidade nos âmbitos local e global trouxe assuntos ligados ao acesso universal à informação e ao conhecimento, à construção social, cultural, à globalização, à garantia cultural, à aprendizagem nas organizações e às questões semióticas da cultura. 
Por fim, ainda que desviando das abordagens tradicionais da organização do conhecimento, o subtema organização da informação para gestão do conhecimento abordou os assuntos: dimensões institucionais da área arquivística, universo da saúde representação em arquivística, arquivos em instituições de saúde, competência profissional para tomada de decisão, realidade dos documentos digitais, estudo de usuário e aprendizagem organizacional.

No evento NASKO de 2011 não houve tema definido sendo que no NASKO 2013 trabalhouse com o tema "Culturas de transição, $O C$ de transição: envolvendo exploração, reflexão crítica e trabalho prático", totalizando 42 trabalhos em ambos os eventos. Esses dois eventos revelaram os seguintes subtemas com os respectivos assuntos:

a) Instrumentos de OC (Linguagens de indexação). Os principais assuntos desse subtema foram: sistemas, ambiências, aplicações, RDA, Taxonomias e vocabulários controlados.

b) Processos de OC, cujos principais assuntos foram: ensino, objetivos e gêneros textuais.

c) Produtos de OC, com os assuntos: catálogos e metadados.

d) Teorias e metodologias de OC, com assuntos tais como: classificação facetada, indexação sistemática, teoria prototípica, análise de domínio e categorização cognitiva.

e) Aspectos culturais, onde destacaram os assuntos: diversidade, multidimensionalidade, bias, construção discursiva, vocabulários emergentes, etiquetagem social e compartilhamento do conhecimento.

f) Interdisciplinaridades em OC, com os seguintes assuntos: diplomática e lógica.

g) Conceitos de OC, com os assuntos: autoria e consistência.

\section{Análise e discussão dos dados}

Relativamente às temáticas, os eventos brasileiros centraram-se nos desafios e perspectivas científicas da OC em um contexto de complexidade, abordando-os sob as dimensões epistemológica, aplicada e sócio-político-cultural.

No caso espanhol, teve-se, em um primeiro momento, o resgate da própria trajetória científica da área na Espanha (história e produção científica), a configuração epistemológica da área como um todo, e sua complexidade disciplinar, para, em um segundo momento, já em um âmbito ibérico, discutir as relações (de identidade e de dissonância) entre informação e conhecimento, a partir de seus fundamentos, modelos e métodos, tendo em vista as necessidades de compartilhamento, e as dualidades entre o local e o global e entre a igualdade e a diversidade.

A NASKO, por sua vez, passou a revelar um tema apenas em 2013, quando centrou-se nas Culturas de transição, levando a uma OC de transição.

Esse cenário, como um todo, revela, de forma geral, aspectos significativos como a necessidade de constante construção epistemológica da área e a sua crescente complexidade, em especial a partir de culturas de transição. Após a identificação dos assuntos percebeu-se que os predominantemente comuns foram: epistemologia, análise de domínio, processos e linguagens de indexação (incluindo a classificação). Os assuntos que assumem uma configuração mais regional em suas aplicabilidades $e$ abordagens foram: resumos e engenharia do conhecimento.

Além dos temas, subtemas e assuntos, foi identificado um total de 340 autores. Posteriormente, foram selecionados os autores com 3 ou mais trabalhos apresentados, o que totalizou 34 autores, ou seja, $10 \%$ do total, que estiveram a cargo de 140 trabalhos selecionados, como se pode ver no Apêndice.

Relativamente ao grau de interlocução nesse espaço científico, observa-se que Guimarães, Martínez-Ávila, Olson, Kipp, Moraes e Sales, respectivamente do Brasil e dos Estados Unidos, encontram-se presentes nos três entornos analisados, o que representa $17 \%$ do universo analisado. Por outro lado, apenas 3 autores, respectivamente da Espanha, do Brasil e da Hungria, encontram-se presentes em apenas um espaço ( $8 \%$ do universo analisado), o que reflete que a grande maioria dos autores selecionados $(75 \%)$ transita por ao menos dois espaços.

Em termos de procedência geográfica dos autores verificou-se que $68 \%$ provêm do Brasil, $15 \%$ da conjunção ibérica (Espanha e Portugal) e $12 \%$ dos Estados Unidos. Sobre essa procedência, destacam-se, institucionalmente, a Universidade Estadual Paulista - UNESP, do Brasil, com $31 \%$ do total, a University of Wisconsin Milwaukee - UWM, dos Estados Unidos, com $11 \%$ do total, e a Universidad Carlos III de Madrid - UC3M, da Espanha, com $10 \%$ do total, sendo que apenas essas três universidades respondem por $52 \%$ da produção analisada, e em forte grau de dialogicidade, haja vista sua colaboração em trabalhos. 


\begin{tabular}{|c|c|c|c|c|c|c|}
\hline Autor & País & Instituição & 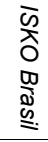 & 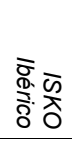 & 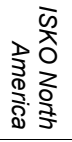 & $\stackrel{-1}{\underline{Q}}$ \\
\hline Guimarães & Brasil & UNEspanha & 04 & 05 & 03 & 12 \\
\hline Fujita & Brasil & UNEspanha & 02 & 04 & & 06 \\
\hline Martínez-Ávila & Espanha & UC3M & 02 & 03 & 01 & 06 \\
\hline Olson & USA & UWM & 03 & 01 & 02 & 06 \\
\hline Moura & Brasil & UFMG & 04 & 01 & -- & 05 \\
\hline Varela-Varela & Brasil & UFBA & 02 & 03 & -- & 05 \\
\hline Agustín. & Espanha & UNIZAR & 04 & -- & -- & 04 \\
\hline Almeida & Brasil & UNEspanha & -- & 04 & -- & 04 \\
\hline Barbosa & Brasil & UFBA & 01 & 03 & -- & 04 \\
\hline Campos & Brasil & UFF & 03 & 01 & & 04 \\
\hline Kipp & USA & UWM & 01 & 01 & 02 & 04 \\
\hline Kobashi & Brasil & USP & 02 & 02 & -- & 04 \\
\hline Lara & Brasil & USP & 01 & 03 & -- & 04 \\
\hline Lima & Brasil & UFMG & 01 & 03 & -- & 04 \\
\hline Milani & Brasil & UNEspanha & 01 & 01 & 02 & 04 \\
\hline Moraes & Brasil & UNEspanha & 01 & 02 & 01 & 04 \\
\hline Orrico & Brasil & UNIRIO & 02 & 02 & -- & 04 \\
\hline Sales & Brasil & UFF & 01 & 02 & 01 & 04 \\
\hline San Segundo & Espanha & UC3M & 02 & 02 & -- & 04 \\
\hline Silva & Brasil & UNIRIO & 02 & 02 & -- & 04 \\
\hline Smiraglia & EUA & UWM & 01 & -- & 03 & 04 \\
\hline Tennis & EUA & UW & -- & 01 & 03 & 04 \\
\hline Barat & Hungria & USzeged & -- & 03 & -- & 03 \\
\hline Barite & URU & UDELAR & 02 & 01 & -- & 03 \\
\hline Barros & Brasil & UNEspanha & -- & 02 & 01 & 03 \\
\hline Fdez-Molina & Espanha & UGR & 02 & 01 & -- & 03 \\
\hline Guedes & Brasil & UFRJ & 01 & 02 & -- & 03 \\
\hline Marcondes & Brasil & UFF & 02 & 01 & -- & 03 \\
\hline Oliveira & Brasil & UNEspanha & 02 & 01 & -- & 03 \\
\hline Ribeiro & Portugal & U.Porto & 01 & 02 & -- & 03 \\
\hline Santos & Brasil & USP & 01 & 02 & -- & 03 \\
\hline Santos & Brasil & UFRJ & 01 & 02 & -- & 03 \\
\hline Talamo & Brasil & USP & 02 & 01 & -- & 03 \\
\hline Tognoli & Brasil & UNEspanha & -- & 02 & 01 & 03 \\
\hline TOTAL & & & 54 & 66 & 20 & 140 \\
\hline
\end{tabular}

Tabela I. Autores mais produtivos e sua presença nos espaços analisados

\section{Considerações finais}

A vista do exposto pode-se concluir que os espaços brasileiro, ibérico e norte-americano da ISKO vêm desenvolvendo uma trajetória de produção científica efetiva, com pontos temáticos de convergência, como as questões epis- temológicas, por exemplo, sem deixar de lado as preocupações com aspectos de ordem mais regional. Nesse contexto, a presença de vários pesquisadores em mais de um dos espaços (ou nos três) revela uma preocupação - e um compromisso com a interlocução acadêmica em organização do conhecimento, destacando-se, institucionalmente, a Universidade Estadual Paulista - UNESP, do Brasil, a University of Wisconsin Milwaukee - UWM, dos Estados Unidos, e a Universidad Carlos III de Madrid UC3M, da Espanha que, inclusive, vêm intensificando sua colaboração científica.

\section{Notas}

(1) Apenas os anais do segundo congresso da ISKO, realizado na Índia, em 1992, não foram publicados na série Advances in Knowledge Organization.

\section{Referências}

Barité, M. (2001). Organización del conocimiento: un nuevo marco teórico-conceptual en Bibliotecología y Documentación. // Carrara, K. (ed.). Educação, universidade e pesquisa. Marília: Unesp, 2001. 35-50.

Bates, M. J. (2005). An introduction to metatheories, theories and models. // Fisher, K. E.; Erdelez, S.; Mckechnie, L. (eds.). Theories of information behavior. New Jersey: Medford, 2005. 1-24.

Broughton, V. (2008). Henry Evelyn Bliss - the other immortal, or a prophet without honour? // Journal of Librarianship and Information Science. 40:1 (March 2008) 45-58.

Dahlberg, I. (1993). Knowledge organization: its scope and possibilities. // Knowledge Organization. 20:4 (1993) 211-219.

Esteban Navarro, M. A.; García Marco, F. J. (1995). Las primeras jornadas sobre organización del conocimiento: organización del conocimiento e información científica. // Scire. 1:1 (Enero/Junio 1995) 149-157.

Garcia Marco, F. J. (1997). Avances en Organización del Conocimiento en España: los II Encuentros sobre Organización del Conocimiento en sistemas de información y documentación. // Garcia Marco, F. J. (ed.). Organización del conocimiento en sistemas de información y documentación. Zaragoza: Universidad, 1997. 7-12.

García Marco, F. J. (ed.) (1997). Organización del conocimiento en sistemas de información y documentación. Zaragoza: ISKO, 1995. 99-112.

Guimarães, J. A. C. (2001). Perspectivas de ensino e pesquisa em organização do conhecimento em cursos de Biblioteconomia: uma reflexão. // Carrara, K. (ed.). Educação, universidade e pesquisa. Marília: Unesp, 2001. 61-72.

Hjørland, B.; Albrechtsen, H. (1995). Toward a new horizon in information science domain analysis. // Journal of the American Society for Information Science. 46:6 (1995) 400-425.

Hjørland, B. (2004). Domain analysis: a socio-cognitive orientation for Information Science research. // Bulletin of the American Society for Information Science and Technology. 30:3 (feb./mar. 2004). http://www.asis.org/Bul letin/Feb-04/hjorland.html (2014-06-23).

Hjørland, B. (2002). Domain analysis in information science: eleven approaches - tradional as well as innovative. /l Journal of Documentation. 58:4 (2002) 422- 462. 
Hjørland, B. (1998). Theory and metatheory of information science: a new interpretation. // Journal of Documentation. 54:5 (1998) 606-621.

Kerr, E. S. (2003). Ketib: um processo de representação de informações para textos complexos. Campinas: Unicamp, 2003. Dissertação de Mestrado Profissional em Ciência da Computação. http://libdigi.unicamp.br/docu ment/?code=vtls000311557 (2014-06-23).

Lloréns, J. et al. (2004). Automatic generation of domain representations using thesaurus structures. // Journal of the American Society for Information Science and Technology. 55:10 (2004) 846-858.

Mai, J.-E. (2005). Analysis in indexing: document and domain centered approaches. // Information processing and management. 41:3 (May 2005) 599-661.

Miranda, M. L. C. (1999). A organização do conhecimento e seus paradigmas científicos: algumas questões epistemológicas. // Informare. 5:2 (1999) 64-77.

Moya-Anegón, F.; Herrero-Solana, V. (2001). Análisis de dominio de la revista mexicana de investigación bibliotecológica. // Información, Cultura y Sociedad. 5 (2001) 10-28.

Pritchard, A.; Groos, O. V. (1969). Documentation notes. // Journal of Documentation. 25:4 (1969) 344-349.
Ritzer, G. (1991). Metatheorizing in Sociology. New York: Lexington Books, 1991. 1-21.

Smiraglia, R. P. (2011). Domain coherence within Knowledge Organization: people, interacting theoretically, across geopolitical and cultural boundaries. // Mckenzie, P.; Johnson, K.; Stevens, S. (eds.). Exploring interactions of people, places and information. New Brunswick: University of New Brunswick, 2011. 6.

Tennis, J. T. (2008). Epistemology, theory and methodology in Knowledge Organization: toward a classification, metatheory, and research framework. // Knowledge Organization. 35:2-3 (2008) 160-182.

Tennis, J. T. (2003). Two axes of domain analysis. // Knowledge Organization. 30:3/4 (2003) 191-195.

Thellefsen, T. L.; Thellefsen, M. M. (2004). Pragmatic semiotics and knowledge organization. // Knowledge Organization. 31:3 (2004) 177-187.

Vickery, B. C. (1997). Metatheory and Information Science. /I Journal of Documentation. 53:5 (1997) 547-476.

Enviado: 2014-04-20. Segunda versión: 2014-06-27. Aceptado: 2014-06-27. 
